\title{
Multiple choice questions: ANSWERS
}

Chapter 1. Overview of stereotactic body radiation therapy for spinal metastasis

1. What is the 1-year local control rate of spine radiosurgery for reirradiation of spine metastases?

$75 \%$

2. What are the three factors used to discriminate classes 1-3 in a recursive partitioning analysis classification scheme for patients undergoing spine radiosurgery?

a. Karnofsky Performance Status (KPS), age and time from diagnosis of primary cancer

How frequent is adjacent vertebral body failure in spine radiosurgery?

$$
\leq 10 \%
$$

What is the most appropriate clinical scenario for delivering spine stereotactic body radiation therapy?

Retreatment of vertebrae previously irradiated with conventional radiation therapy

How frequently does vertebral compression fracture occur in spine stereotactic body radiation therapy?

$$
10-15 \%
$$

Chapter 2. Immobilization, target delineation and contouring of organs at risk

What modality should be used to delineate the spinal cord when a patient cannot undergo a spinal MRI?

CT myelogram 
2. Which one of the following is the most appropriate planning treatment volume expansion for a spinal metastasis?

$3 \mathrm{~mm}$

Which of the following is the most appropriate planning organsat-risk volume for the spinal cord for spinal stereotactic body radiation therapy?

Thecal sac

What is the mean motion range of the spinal cord at the thoracic level?

$0.5 \mathrm{~mm}$

Which one of the following is the most appropriate clinical target volume for a centrally located $1-\mathrm{cm}$ spinal metastasis occurring in the anterior portion of T5, approximately $1 \mathrm{~cm}$ from the cortex?

Whole vertebral body

Chapter 3. Treatment planning, intrafraction monitoring, and delivery: linear accelerator-based stereotactic spine radiotherapy

True or false? Linear accelerator (LINAC)-based stereotactic spine radiotherapy is clearly superior to high-dose conventionally fractionated radiotherapy also delivered with a LINAC?

False

2. True or false? The clinical outcomes with conventional LINAC-based spine stereotactic body radiation therapy (SBRT) are inferior to the same treatment delivered with other techniques or technologies.

False

True or false? Spine SBRT requires a LINAC that is set up and maintained for high-precision treatment delivery.

True

True or false? Treatment plans made with conventional intensitymodulated radiotherapy are inferior to newer delivery methods such as volumetric modulated arc therapy.

False 
True or false? Near-rigid external immobilization guarantees patients, or their spine, will not move during treatment.

False

True or false? High-dose rate delivery is inherently superior to treatment using slower dose rates.

False

\section{Chapter 4. Treatment planning, intrafractional tracking and deliv- ery: CyberKnife ${ }^{\circledR}$-based stereotactic body radiation therapy}

The CyberKnife ${ }^{\circledR}$ (CK; Accuray Inc., CA, USA) system allows for increased accuracy in spine stereotactic body radiation therapy (SBRT) because it...

Has the capacity to minimize the residual target motion that could result in increased spinal cord dose by using frequent imaging of the target position

2. Which of the following are true regarding patient simulation for CK spine treatments (may have more than one correct answer)?

The CT scan should be centered on the target and should include at least $10-15 \mathrm{~cm}$ above and below the target volume

e. Patient comfort should be a major consideration

The following could be expected for CK spine SBRT plans:

There could be hotspots as large as $130 \%$ of the prescription dose

What is true regarding intrafractional tracking and treatment delivery in CK?

CT slice thickness can influence the tracking accuracy

According to Radiation Therapy Oncology Group 0631: "A Phase II/III study of image-guided radiosurgery/SBRT for localized spine metastasis" [102]:

The prescription dose could be either 16 or $18 \mathrm{~Gy}$ 


\section{Chapter 5. Clinical applications of stereotactic body radiation therapy: primary and postoperative settings}

Spinal stereotactic body radiation therapy (SBRT) should not be utilized for patients with:

a. An unstable spine

2. Spinal SBRT generally affords all of the following except:

Restoration of vertebral body height

Spinal SBRT is generally effective for radioresistant tumor histologies.

True

Spinal SBRT cannot be safely administered after prior fractionated radiotherapy.

False

What is the most common indication for spinal SBRT?

Metastasis

A patient with a spinal metastasis successfully treated with spinal SBRT begins to develop increasing back pain at the level of the treatment. The most likely cause of this new onset of back pain is:

A compression fracture

Chapter 6 . Reirradiation of spinal metastases with spine stereotactic body radiation therapy

What is the most essential aspect of safe stereotactic body radiation therapy (SBRT) treatment delivery?

All of the above

2. In terms of SBRT doses, literature states that higher doses:

None of the above

The main pattern of failure after reirradiation is:

Epidural disease

Overall survival of patients treated with reirradiation is influenced by:

All of the above

The risk of vertebral compression fracture is dependent on:

Dose per fraction 


\section{Chapter 7. Clinical applications: epidural spinal cord compression}

What is the standard treatment for previously unirradiated epidural spinal cord compression from spinal metastasis?

Surgical decompression followed by postoperative radiotherapy

2. What can generalizable class solutions achieve?

All of the above

Which one of the following is an absolute contraindication to SBRT for epidural spinal compression?

Patients with motor power of four out of five in the lower extremities

Which one of the following is the biggest challenge to the use of SBRT for patients with epidural spinal cord compression from spinal metastasis?

Necessity for prompt treatment planning and quality assurance

Which one of the following is correct regarding immobilization for spinal SBRT?

A stereotactic bodyframe with vacuum wrap has been shown to provide the most robust immobilization

\section{Chapter 8. Optimal fractionation for SBRT for spinal metastasis}

What is the recommended single-fraction dose in the stereotactic body radiation therapy (SBRT) arm of RTOG 0631?

16 Gy

Which dose is associated with increased local control in the Memorial Sloan-Kettering Cancer Center spine SBRT series (Yamada et al. [2008] [13])?

$$
24 \mathrm{~Gy}
$$

What fractional dose is associated with and a six- to seven-fold increase in risk of vertebral compression fractures (Cunha et al. [2012] [21])?

$$
>20 \text { Gy }
$$


A potential advantage of single- versus multi-fraction spine SBRT is:

Decreased cost

\section{Chapter 9. Radiation myelopathy and spinal cord tolerance}

According to the literature on human spinal cord tolerance, the spinal cord is a:

Serial organ

2. The spinal cord is a static organ:

False

For spine stereotactic body radiation therapy (SBRT) treatment, experts recommend to contour:

The thecal sac or cord on MR images fused to the planning

CT-scan or on a planning CT myelogram

Intrafraction motions are more susceptible to occur in the:

Cervical spine

For single-fraction stereotactic radiotherapy and no prior radiation, for a probability of $5 \%$ or less of radiation myelopathy, the thecal sac $P_{\text {max }}$ dose should not exceed:

12.4 Gy

Chapter 11. Other complications associated with spinal stereotactic body radiation therapy

Which one of the following is not a risk factor for vertebral compression fracture after stereotactic body radiation therapy (SBRT) for spinal metastasis?

Renal cell carcinoma primary

Which of the following regarding pain flare is correct?

Steroid therapy can reduce the risk of pain flare after SBRT for spinal metastasis

Regarding esophageal toxicity, which of the following is correct?

The incidence of grade 3 or higher acute or late esophageal toxicities is $<10 \%$

What is the tolerance of the brachial plexus to SBRT?

24-26 Gy in three to four fractions 
Regarding SBRT-induced radiculopathy/plexopathy, which of the following statements is correct?

There are likely to be residual neurologic deficits even after treatment of SBRT-induced radiculopathy/plexopathy

\section{Chapter 12. Histopathological examination of spine metastases after radiosurgery}

Which of the following histopathological changes may be observed in spine metastases specimens after stereotactic body radiation therapy (SBRT)?

All of the above

2. Which of the following statements regarding histopathological changes in spine metastases after SBRT is correct?

Viable tumor is not present in all cases of symptomatic compression fractures

Which of the following statements regarding the presence of tumor within the gross tumor volume (GTV) is true?

Tumor presence within GTV does not necessarily indicate disease progression

Surgical intervention after SBRT for spinal metastases is:

Both $b$ and $c$.

Which of the following statements regarding histopathological examination of spine metastases after SBRT is true?

Histopathological examination of spinal metastases after SBRT should be performed when undergoing subsequent surgery 